\title{
A Research on Music Therapy Intervention in the Anxiety of Families of Dying Patients
}

\author{
Yawei Yu, Ning Qu* \\ College of Humanities, Jiangxi University of Traditional Chinese Medicine, Nanchang Jiangxi, 330000, China \\ *Corresponding author
}

Keywords: music; family members of dying patients; emotional problems.

\begin{abstract}
To help the families of dying patients (subjects) reduce their fear and anxiety due to the death of their relatives, enhance their self-concept, promote relaxation, relieve stress and reduce anxiety. On the one hand, the subjects were interviewed to learn about the basic state of psychological crisis. On the other hand, SAS scale was used to evaluate the anxiety state of 30 subjects and randomly divide them into an experimental group and a control group. The means and methods of music therapy were used to intervene in the emotional state of the family members of the dying patients in the experimental group. The results of SAS pre-testing and post testing were statistically analyzed after the experiment. About music therapy intervention in the anxiety of the families of dying patients, the anxiety state of the experimental group is significantly reduced compared with the control group, $\mathrm{P}<0.05$. The use of various forms and functions of music, which can significantly improve the anxiety state of dying patients, is a good intervention means for psychological crisis.
\end{abstract}

\section{Introduction}

The fact of being dying does not only to bring a heavy physical and psychological burden to the dying patient, but also brings great psychological stimulation to the families. During the fight between the dying patient and the disease, the family members have to both shoulder enormous financial pressure and suffer the pain caused by the imminent death of their relatives. Under the severe economic and spiritual pressure, persistent unhealthy factors will exceed the scope of the body's regulation, resulting in functional disorder and disorder of qi and blood. Most of the family members of dying patients have abnormal emotional performance, such as anxiety, depression, and insomnia, etc. Troubled by the long-term emotional problems, family members of the dying patients will have serious psychological problems, some of which may even lead to over-aggressive behaviors, conflict between doctors and patients, and even worse, bloodshed, threatening social stability.

Music can change the blood pressure, respiration and heart rate, increase the number of immunoglobulin in the body, improve the physiological function, relieve the tension and anxiety obviously, and help people relax.

\section{The Basic Situation of Subjects}

Inclusion criteria: (1) those whose relative(s) will die in a few weeks to months by the medical diagnosis; (2) those who fit the diagnostic criteria for anxiety after the interview and the SAS scale assessment; (3) those who are interested in music or not exclusive.

Exclusion criteria: (1) those who do not want to participate in music therapy activities or are not willing to accept random distribution; (2) those who have mental illnesses; (3) those who suffer hearing loss, speech impairment and cognitive impairment.

After screening through the above conditions, 30 family members of dying patients from the hospice center in a hospital were selected and randomly divided into the experimental group and control group with 15 people each, including 13 males and 17 females; the youngest was 19 years old 
and the oldest was 67 years old; none of them has a professional musical background.

\section{The Experimental Research}

\subsection{The Research Process}

The research was divided into three stages. First of all, the subjects were interviewed, assessed with the SAS scale, and then randomly divided into two groups. According to the evaluation results of the pretesting and the interviews, the targeted personalized music therapy interventions were conducted on the state of psychological crisis in the experimental group, while there was no intervention in the control group. Music therapy interventions were conducted twice a week, for about 40-60 minutes each time according to the state of the subjects. Both groups were reevaluated after 16 musical interventions.

\subsection{Technical Methods}

The preferences for music and physical state of mind of the family members of dying patients were learned about through interviews to develop targeted personalized music therapy programs for each one of them. The following technical methods were mainly used:

(1)Gradual relaxation of music: Many types of music suitable for relaxation for the subjects were provided to be chosen, or the subjects prepared music that made them feel relaxed, which the therapist assessed to decide whether it met the requirements of relaxing music. Music and relaxation guide language were combined so that the subjects had relaxed muscles, steady breath, slow heart rate, reduced anxiety and eased sleep disorders.

(2)Systematic desensitization of music: Some subjects were anxious in specific scenes or situations. Systematic desensitization was used with music to desensitize the stimuli and help to reduce the anxiety reaction to the stimuli.

(3) Musical psychodrama: When the relatives are about to or have passed away, subjects expressed their inner feelings through the techniques of musical psychodrama, changed their cognition with the help of music therapists and established the correct idea of treating death.

(4) A safety island: During the treatment, the subjects would find it hard to control their emotions or have great mood swings, which was bad for the continuation of the treatment. At this time, it is very important for the subjects to seek inner safety and stability.

(5) Musical composition: The use of musical composition could not only maintain the emotional relationship between patients and their families, but also show the grief to the passed ones.

\subsection{Research Tools}

\subsubsection{Psychological Tools}

According to the research needs, an interview questionnaire was self-made, which was divided into two parts. Part One is an investigation of the subjects' basic conditions, including age, gender, hobbies, music background and other related issues; Part Two is an investigation of the subjects' mental and physical symptoms, including emotions, energy, memory, sleep, suicidal tendencies and so on.

The SAS anxiety self-rating scale is used as a psychological scale to measure the severity of anxiety and its changes during treatment, which is one of the commonly used psychological measurement tools, with a total of 20 four-grade scores. The answer should be in accordance with the actual situation of the last week, and converted into standard scores. The higher the score was, the more serious the symptoms were.

\subsubsection{Therapeutic musical instruments}

Musical audio equipment, pianos, Ukuleles, xylophones, glockenspiels and various types of drums. 


\subsection{Statistical processing}

SPSS17.0 statistical software is used in statistical analysis, and each group of data is expressed with $\bar{x} \pm s$. T test analysis of paired samples is conducted in the control group and experimental group.

\section{Result}

\subsection{The Interview}

Interviews and questionnaires show that patients have a certain degree of receptivity and aesthetic ability in music without rejection, and have a preference for music related to the important stages of their life. In addition, in the physiological aspect, some of the subjects have symptoms such as physical fatigue, headache and severe insomnia that can trigger anxiety due to long-term care of patients. In the psychological aspect, there is a misconception of the end of life and death itself, which leads to a great sense of fear. Other misconceptions can also bring guilt, self-blame, and feelings of powerlessness to the subjects. The economic pressure and the reduction of social interaction will also bring much psychological pressure to subjects, which will lead to anxiety.

\subsection{Comparison of the SAS pre-testing and post testing}

Table 1. T test inspection of paired samples of pre-testing and post-testing in the SAS control group and intervention group

\begin{tabular}{|c|c|c|c|c|c|}
\hline Groups & Stages & Examples & $\bar{x} \pm s$ & $\mathrm{~T}$ & $\mathrm{P}$ \\
\hline \multirow{2}{*}{ Control group } & $\begin{array}{c}\text { Before } \\
\text { intervention }\end{array}$ & 15 & $47.20 \pm 3.28$ & \multirow{2}{*}{0.86} & 0.40 \\
\cline { 2 - 4 } & $\begin{array}{c}\text { After } \\
\text { intervention }\end{array}$ & 15 & $46.87 \pm 3.09$ & & \multirow{2}{*}{$0.00^{* *}$} \\
\hline \multirow{2}{*}{$\begin{array}{c}\text { Intervention } \\
\text { group }\end{array}$} & $\begin{array}{c}\text { Before } \\
\text { intervention }\end{array}$ & 15 & $47.01 \pm 3.17$ & \multirow{2}{*}{ After } \\
\cline { 2 - 4 } & intervention & 15 & $30.73 \pm 2.15$ & 16.94 & \\
\hline
\end{tabular}

Note: $* * \mathrm{P}<0.01$

It can be seen from the data in the table that the SAS in the control group before and after the intervention has no significant difference, while the intervention group before and after the intervention has significant difference $(\mathrm{P}<0.01)$, indicating that interventions have a good effect on the improvement of the subjects' anxiety.

\section{Discussion and conclusion}

Psychological crisis refers to the psychological reaction of an individual when he cannot evade or use their own resources and stress modes to deal with emergencies, major setbacks or difficulties. The death of a relative is the most common psychological crisis. Psychological crisis intervention refers to the definite and effective measures taken to help an individual who is in a state of psychological crisis overcome the crisis and adapt to life again.

From the research, we can find that both the active participation in and passive listening to the music therapy alleviate the anxiety of the families of dying patients. Of course, we also find that some objective reasons, such as the sudden deterioration of the patient's condition, etc., will cause the relapse of anxiety, which affects the treatment result.

In the hospice institutions of some developed countries, there are always music therapists working at appropriate psychological assistance to individuals in a state of crisis with music so that they can get out of trouble as soon as possible, which is a common intervention means. For example, Teresa, a music therapist at St. Patrick's Hospital in Missoula, Montana, USA, developed the "Sabbath of Rest" 
program, which she described as "Music Thanatology", and developed basic hospice projects at the Nursing Center. In foreign hospice care centers, music has become the most popular adjunctive therapy in hospice services to help reduce the fear and anxiety of the families of dying patients, enhance their self-concept, promote relaxation, alleviate sleep problems and reduce anxiety and depression, and reduce loneliness and anger. While engaging in music activities, not only are the emotional vitality and emotional support enhanced, but also dying patients can communicate with their families in the way of music to soothe their sorrows.

Music is a powerful tool to influence people's emotions. The music method helps family members of dying patients alleviate the fear and anxiety of their death, enhance self-concept, promote relaxation, relieve stress, solve sleep problems and reduce anxiety and depression, which fully reflects the need of humane care and the unity of quality and value of life. In this research, music intervention in the psychological crisis of the family members of dying patients was conducted to enrich the hospice care services, enhance the effectiveness of hospice care services, promote the development of the discipline, lay a theoretical foundation for the future study on music therapy technology in this area, provide an effective basis for the clinical practice of music therapy in our country and has important theoretical and practical value.

\section{Acknowledgement}

This article is the result of "Research on Music Intervention in Mental Crisis of dying patient's Families" (YG2016117), and the result of the Jiangxi Humanities and Social Science Project "Research on Hospice Care under the Perspective of Music Psychology" (JC152002 ); Part of the fund of this article is from the key cultivated discipline "Psychology of TCM" of the State Administration of Traditional Chinese Medicine and Jiangxi Culture and Art Science Key Research Base "Gan Po TCM Cultural Psychology Research Center".

\section{References}

[1] S. Munro \& B. Mount. Music therapy in palliative care. Can Med Assoc, 1978 November 4; 119(9): 1029-1034.

[2] Qu Ning. Social Survey Research on Music Therapy in the Field of Hospice in Our Country, Chinese Public Health,2016.6.

[3] Li Yiting, Li Wei. Theory of Hospice Care, Beijing: China Science and Technology Press. Edition 2003.

[4] Qu Ning, Duan Senhai. Study on the Status Quo of Music therapy in the Field of Hospice in Our Country, Technology Wind, 2015.09.

[5] Qu Ning, Zeng Qingxia, Ren Yuan, etc. Clinical Study on the Alleviation Degree of Group Music Therapy for the Psychological Pain of Dying Patients with Terminal Cancer, Human Health, 2016,02. 